\title{
Classification and ways of China urban express terminal distribution service modes
}

\author{
Wang Shengjin, Chen Ying* \\ Shanghai Urban Construction Vocational College, Shanghai, 201415, China
}

\begin{abstract}
In order to meet the diversified consumption needs of urban residents, many new urban express terminal distribution service models have emerged, from the traditional "door to door" service to the current service station and smart cabinets and other service modes coexist. The diversification of urban express delivery service models can not only bring customers a better consumer experience, but also help to further improve the consumption level of urban residents. Through qualitative comparison and analysis of existing urban express terminal distribution service models, this paper can provide a reference for e-commerce companies and logistics companies to choose the urban express terminal distribution service models suitable for their own development. Improving the current status of urban express delivery services will help resolve the contradiction between customer experience demands and enterprises, bring economic benefits to enterprises, and also help improve urban transportation and bring social benefits.
\end{abstract}

\section{Introduction}

The development of my country's e-commerce started at the end of the 20th century. Although it started late compared to developed countries, the momentum of development should not be underestimated. Especially since entering the 21 st century, with the popularization of the Internet, e-commerce has penetrated into all aspects of people's lives at an alarming rate. E-commerce began to show a vigorous, healthy and stable development trend. However, many troubles followed, such as the creation of network technology, credit system issues, logistics and distribution, and laws and regulations. The most important one is the construction of logistics distribution. As a carrier supporting the entire online business activities, logistics distribution is not only an obstacle to online tangible commodity transactions, but also a key factor in the success of online related commodity transactions. Without a sound and effective logistics distribution system, it will be difficult for e-commerce to take advantage of its advantages. Especially in e-commerce transactions, online shopping is not restricted by time and region. Online shopping users are relatively scattered, so the requirements for logistics and distribution are even higher. The purpose of logistics distribution is to deliver items to customers. Therefore, in the entire logistics distribution process, the express terminal distribution service is the top priority. If logistics and distribution are the bottleneck restricting the development of e-commerce, then terminal distribution services are the bottleneck restricting the development of logistics and distribution. Therefore, it is particularly important to study the end-of- city express delivery service mode and analyse how to choose the end-of-city express delivery service mode.

\section{Classification of end-of-city express delivery service models}

This article uses the enterprise's operation mode and service mode as the division standard to construct the city express terminal distribution service mode.

\subsection{Vancl Rufengda mode}

The Vancl Rufengda model is that the distribution company builds its own logistics distribution system, and does not set up a service station at the end. The employees of the distribution company directly deliver the customer packages to the customers. Vancl's self-built logistics distribution "Rufengda" in April 2008 was acquired by Tiandi Huayu Group in 2014, but it is a typical direct selfoperated service model. This is named the Vancl Rufengda model. In addition, JD.com, Suning Appliance and Yihaodian in some places also use this model for city express terminal delivery services.

\subsection{Easy housework mode}

The easy-to-family model is that the distribution company sets up a smart cabinet service station at the end, and the employees of the distribution company put the customer's package in the smart cabinet service station, and the customer can pick up the express within the specified time. The Jiashiyi Fresh Supermarket in Wuhan is setting up a smart cabinet service station in the community. After the

e-mail: CJ0840@succ.edu.cn 
customer places an order online, the Jiashiyi Company will deliver the goods in a timely manner, put the goods in the smart cabinet service station, and then the customer picks it up by themselves. As the terminal delivery service model of Wuhan Jiashiyi Company is relatively complete and its operation is relatively mature, it is highly praised by the society. Therefore, this article named the selfoperated smart cabinet model as Jiashiyi mode.

\subsection{SF Express mode}

The SF model is that the distribution company sets up a manual service station at the end. Customers can either pick up their parcels from the manual service station or deliver them to the door by the employees of the distribution company. The SF Express model is the selfoperated express station model that is well known to everyone. In the initial stage of the establishment of SF Express Station, SF Express Company built its own express service station and carried out express delivery, but the packages it delivered were basically packages from other e-commerce companies. However, since 2012, SF Express established its own e-commerce platform-SF Optimum. Some parcels delivered by SF began to come from its own e-commerce platform. In 2014, SF established Heike convenience store in the community, and customers can either request home delivery or you can extract it yourself. As a result, SF Express's model has been further optimized and improved, and has become increasingly mature.

\subsection{Jingdong Daojia mode}

The JD Daojia model is that the distribution company sets up a manual service station at the end. Customers can either pick up their packages at the manual service station by themselves or deliver them to the public. Among them, the public mainly refers to the delivery companies employing social people such as students and square dancers to provide door-to-door service, rather than the company's own employees. JD Daojia.The model is a new terminal delivery service model that only appeared in 2015. In order to better serve customers and achieve the timeliness and high efficiency of terminal delivery services, JD employs aunty square dancers to provide free delivery services at home.

\subsection{Amazon outsourcing mode}

The Amazon outsourcing model means that the delivery company will be outsourced to a third party, and the third party does not set up any express service at the end station and deliver the package directly to the customer. At present, in addition to Amazon. In addition to the construction of logistics, logistics outsourcing is implemented for some more remote areas, for example, in Wuhan, Amazon's logistics outsourcer is Feiyuan Logistics. Feiyuan Logistics is no express service station at the end, and the package is directly delivered to the customer.

\subsection{Express delivery mode}

The express mode is a third-party company set up at the end smart cabinet service station, the distribution company puts customer packages in the smart cabinet service station, and the customer can pick up the package within the specified time. Distribution companies in this article refers to e-commerce companies and express companies, such as express delivery companies established in the end community smart cabinet service station, the distribution company cooperates with the express company to send the parcels in the smart cabinet, according to the number of parcels; Easy Express will allocate different send companies to charge.

\subsection{Fengchao mode}

The Fengchao model is that distribution companies jointly invest in the establishment of a terminal intelligent cabinet service station, and customers can pick up their packages within a specified time. In 2015, SF cooperated with Shentong, Zhongtong, Yunda and other companies to initiate the establishment of Shenzhen Fengchao Technology Co., Ltd. ("Fengchao" for short), which invested in the establishment of smart cabinet service stations at the end.

Table1. Classification of end-of-city express delivery service models

\begin{tabular}{lccccc}
\hline & 2015 & 2016 & 2017 & 2018 & 2019 \\
\hline Express & 233653 & 324848 & 349498 & 412932 & 675775 \\
Domestic & 65588 & 76727 & 83666 & 89003 & 98882 \\
$\begin{array}{l}\text { Non- } \\
\text { domestic }\end{array}$ & 134546 & 233761 & 365711 & 463672 & 565656 \\
\hline
\end{tabular}




\section{Suggestions on improving the end-of- city express delivery service mode}

\subsection{Collaboration of terminal distribution services}

In addition to self-built logistics distribution systems for distribution, enterprises can also cooperate with other organizations for end-of-end distribution, which can improve the end-of-end distribution efficiency and save resources. Currently, distribution companies mainly implement two forms of collaboration. One is to cooperate with schools, communities, chain convenience stores, national government agencies, and large office buildings to establish terminal distribution sites close to consumers and implement terminal smart cabinet services. The other is the cooperation between e-commerce companies, express companies and specialized end-of-end distribution companies to outsource end-of-end delivery to a dedicated third-party delivery party. The third-party delivery party builds its own service station at the end to realize the unified and specialized end-of-end delivery.

\subsection{Standardization of terminal distribution services}

At present, the terminal distribution service lacks unified norms and standards in terms of management and specifications, which are not conducive to the management of urban express terminal distribution services. Therefore, the government should coordinate various departments to conduct dynamic management of terminal distribution, regulate the matching standards of vehicles and personnel for terminal distribution, and realize the standardization and standardization of terminal distribution. Enterprises should also continuously improve their own standardization and standardization in terms of distribution rules and regulations, personnel management, and distribution service facilities and equipment. This can not only save the cost of distribution companies, but also maximize the use value of the entire social resources.

\subsection{Intelligentization of terminal distribution services}

The intelligentization of terminal distribution here mainly refers to the intelligence of terminal distribution facilities. With the continuous increase of labour costs, the cost of end-of-end distribution of distribution companies is also getting higher and higher. Based on this consideration, distribution companies began to establish smart cabinets, service stations and other end-of-end smart distribution sites at the end, which not only greatly reduces the cost of end-of-line distribution, but also reduces traffic congestion and environmental pollution caused by manual delivery of express mail. However, the intelligentization of urban express delivery facilities can only play its role better when they become public facilities. Therefore, the government should adopt relevant and reasonable mechanisms to make the urban express delivery terminal distribution facilities become public facilities invested and established by the government for corporate public use, so as to better play the role of smart facilities.

\subsection{Convenience of terminal distribution service}

The convenience of terminal distribution services here includes two levels: the first level refers to the convenience of services, that is, the flexibility of delivery time, the efficiency of distribution speed, and the sharing of distribution information; the second level refers to terminal distribution the facility is simple and easy to operate. The terminal distribution facilities should be easy to learn and easy to use for the public, so as to improve customer satisfaction and enhance user stickiness. Therefore, the convenience of the terminal distribution service model is a major issue that all distribution companies should consider which has a great impact on the performance of the distribution company.

\subsection{Extensive end distribution services}

The service group of the end distribution determines its extensiveness. Therefore, whoever masters the end distribution will control the customer. Enterprises can establish a terminal distribution service system through self-built terminal distribution systems or cooperation with third parties according to their own conditions and strengths. The extensive coverage of the terminal distribution service network can well integrate terminal consumption resources, expand the enterprise customer base, and create more commercial value. Therefore, the terminal distribution service model should have a certain degree of versatility and can serve all customer groups. Only in this way can the terminal distribution service model truly play its role in serving customers.

\section{References}

1. Yang Juping, Yang Changchun, Yao Xuanxia. (2018) Research on the "Last Kilometer" Problem in Ecommerce Logistics. Business Economics and Management, 14:18-39.

2. Zhang Xin. (2016) The joint distribution mode and decision path of terminal logistics-Analysis of supply and demand based on e-commerce logistics and community service. Research on Financial Issues, 3:39-61.

3. Liu Xiaoning. (2018) The development status and prospects of the terminal logistics model of "Internet + community". Business Economics Research, 17:2237.

4. Wang Yan. (2019) Analysis of the matching degree between community categories and terminal logistics models. Business Economics Research, 17:127-142.

5. Yan Min. (2015) E-commerce logistics value chain analysis based on big data. Business Economics Research, 15:201-236. 